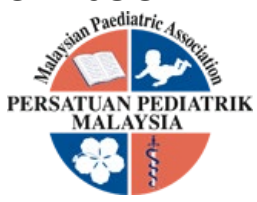

\title{
REVERSAL OF KETAMINE INDUCED ACUTE DELIRIUM WITH CONTINUOUS MIDAZOLAM INFUSION IN A POST EXTUBATION CHILD WITH REFRACTORY BRONCHOSPASM: A CASE REPORT
}

\author{
Fahisham Taib ${ }^{1,3}$, Laila Ab Mukmin 2,3
}

\begin{abstract}
Continuous ketamine infusion has been used successfully to treat severe refractory bronchospasm in children requiring mechanical ventilation in the intensive care setting. One of the common side effects known is emergence delirium. There is no standardized treatment for the ketamine-induced emergence delirium although benzodiazepine, haloperidol and dexmedetomidine have been reported to be effective in the past. A 7-year-old girl admitted to a tertiary hospital for life-threatening asthma requiring immediate intubation and ventilation. Ketamine was used as sedative and bronchodilator in anticipation of her challenging ventilation strategy. She was successfully extubated on day 8 of admission, however, she developed symptoms associated with the delirium. Successful reversal of the symptoms was achieved after 48-hour use of low dose intravenous midazolam. This was the first case reported on the reversal of ketamine-induced emergence phenomenon using low dose intravenous midazolam infusion.
\end{abstract}

Keywords: Ketamine; Delirium; Psychosis; Midazolam; Reversal

\section{Introduction}

The psychotropic effects of ketamine are now well-known ranging from dissociation anaesthesia and schizophrenia-like symptoms. Ketamine-induced emergence phenomena is a side effect characterized by euphoria, vivid dreams, illusions, delirium, hallucinations, sensory distortions, depersonalisation, derealisation, acute severe memory disruption, nausea and vomiting [1]. Continuous ketamine infusion has been used successfully to treat severe refractory bronchospasm in children requiring mechanical ventilation [2]. The incidence of emergence delirium (ED) from ketamine use ranges from 3\% to $100 \%[3,4]$. Haloperidol has been used to reverse ketamine induced emergence delirium in an adult [5]. Benzodiazepine, promethazine, propofol and dexmedetomidine also have been reported to be effective in preventing ketamine induced ED, but these drugs are used to prevent pre surgical procedure [6]. To the best of our knowledge, this is the first time successful use of continuous infusion of midazolam in the
Received: 30 July 2020; Accepted revised manuscript: 27 August 2020

Published online: 09 September 2020

treatment of ketamine induced acute delirium in child with post ventilation for refractory bronchospasm.

Case

A 7-year-old girl was referred to a tertiary centre following a short history of rapid breathing. She has moderate persistent asthma, and her asthma symptoms has been poorly controlled despite being on prophylaxis steroid inhaler. She has history of repeated visit to private clinic for nebulization. On arrival to emergency

\footnotetext{
'Department of Paediatric, School of Medical Sciences, Universiti Sains Malaysia, Kubang Kerian, Kelantan, Malaysia

2Department of Anaesthesiology, School of Medical Sciences, Universiti Sains Malaysia, Kubang Kerian, Kelantan, Malaysia

${ }^{3}$ Hospital Universiti Sains Malaysia, 16150 Kubang Kerian, Kelantan, Malaysia

Corresponding author: Dr. Laila Ab Mukmin,

Department of Anaesthesiology, School of Medical

Sciences, Universiti Sains Malaysia, Kubang Kerian,

Kelantan, Malaysia Tel: 609-767 6313

Email: lailam@usm.my
} 
department, she looked lethargic, unable to speak and has features of severe respiratory distress. She was diagnosed as severe asthma and was immediately intubated.

She was started on regular salbutamol nebulizer initially and added with intravenous magnesium sulphate. Her ventilatory setting was getting worse which warranted additional intravenous ketamine, with initial dose of $1 \mathrm{mg} / \mathrm{kg}$ followed by maintenance dose of $10 \mathrm{mcg} / \mathrm{kg} / \mathrm{hr}(1 \mathrm{ml} / \mathrm{hr}=$ $6.5 \mathrm{mg} / \mathrm{hr}$ ). Her ketamine dose was increased to $2 \mathrm{ml} / \mathrm{hr}(13 \mathrm{mg} / \mathrm{hr})$ due to progressive worsening of her bronchospasm. This step has gradually improved her clinical status. Ketamine was slowly weaned down and stopped on day 6 of admission. She was extubated on day 8 of admission.

Following extubation, her parent reported that she was not being 'herself', restless and agitated. She described of seeing 'ghost-like' figures around her bedside (Figure 1). Patient received a low dose of intravenous midazolam $(0.1 \mathrm{mg} / \mathrm{kg})$ infusion for 2 days, until her delirium symptoms were completely resolved.

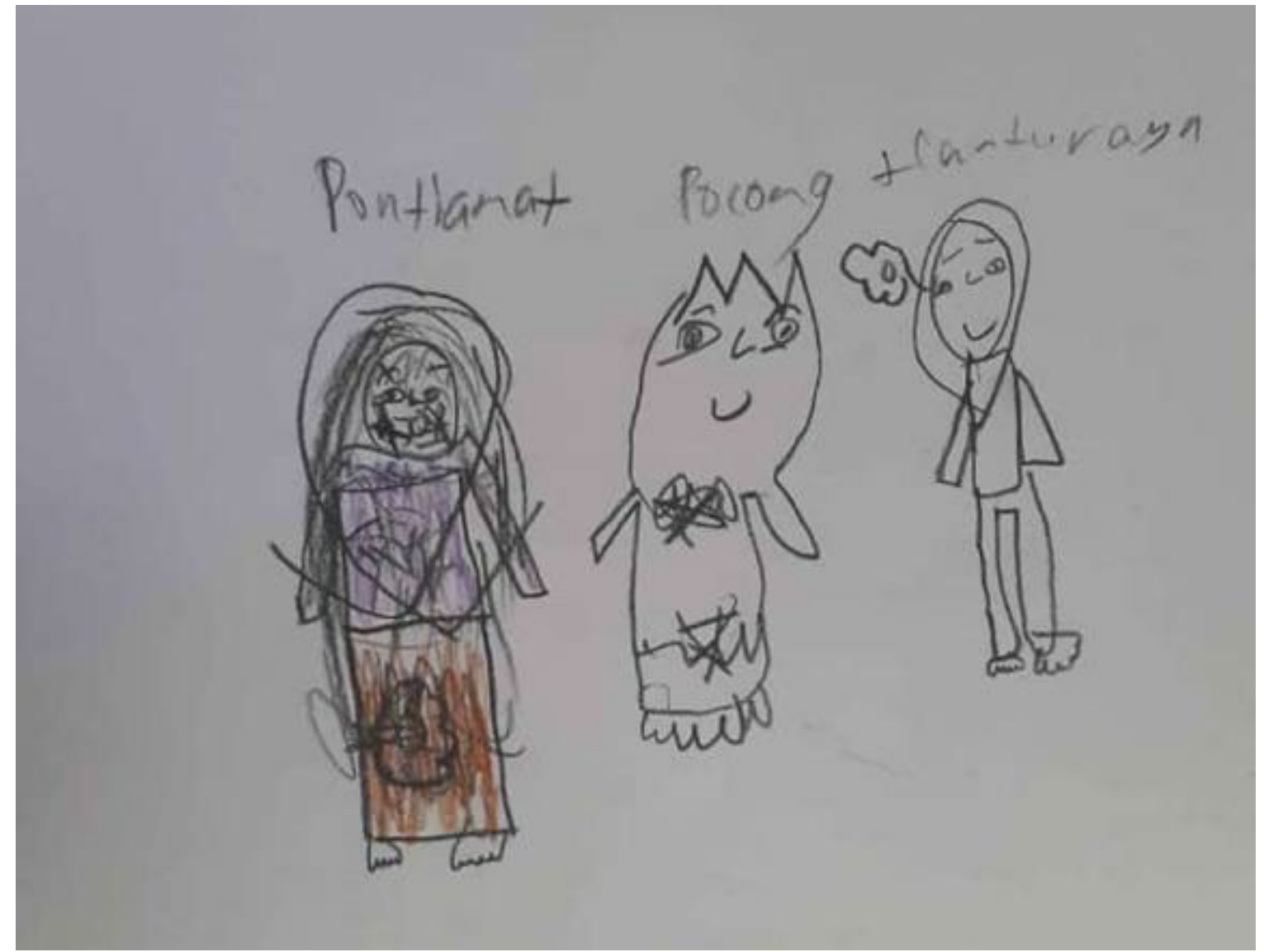

Figure 1. Patient's drawing reported as seeing different ghostlike figures around her

\section{Discussion}

Ketamine has a unique ability to provide analgesia, anti-depressant and anti-inflammatory (7). This is activated via the limbic system by depressing the cerebral cortex glutamate transmission at the $\mathrm{N}$-methyl-D-aspartate receptors (NMDAR). Its non-competitive antagonism of the NMDAR simultaneously blocking the NMDA Ca ${ }^{2+}$ channel pores resulted in sympathomimetic, sedative and bronchodilatory effects. This makes it a preferred anaesthetic drugs in severe asthma. Ketamine also inhibits HCN1 receptors, which causes the hyperpolarisation-activated cation current and thus regulates the states of consciousness and neuropathic pain through inflammatory cascades. 
Mediation of emergence delirium effect is thought to occur from dopamine DA-2 receptor activation. Causes of ketamine related adverse drug reactions are multi-factorial. Genetic polymorphism of the NMDAR gene has been associated with NMDAR hypo-function, psychotic symptoms, and memory disorders (8). Ketamine exerts its clinical effects by non-competitively binding to the NR2 subunit of the NMDAR and blocking the excitatory effects of glutamate. The glutamatergic pathway activation leads to symptoms such as auditory, verbal and visual hallucinations in $60-80 \%$ of the patients [8]. Ketamine increases toxicity level of glutamate and reduce cell viability despite being an NMDA antagonist [9].

Ketamine has different bioavailability depending on the route of administration ranging from 893\%.The onset of action of ketamine is seconds intravenously and longer approximately between 15 to 30 minutes orally. If given orally, ketamine undergoes first-pass metabolism, where it is transformed in the liver by CYP3A4, CYP2B6, CYP2C9 isozymes into norketamine. Norketamine is about one third potent anaesthetic agent. Its duration of action is between 0.5 - 6 hours. Studies have demonstrated inter-individual variability of ketamine pharmacokinetics and pharmacodynamics. Frequently repeated doses of ketamine is associated with prolonged elimination time up to 11 days, and possibly due to overproduction of norketamine in children [10].

As reported in our case study, the patient experienced both negative and positive emergence phenomenon (EP) at a relatively lowdose of ketamine. The description experienced by our patient was related to neuropsychiatry side effects. The symptoms persist for more than 12 hours, far exceeding the half life of ketamine. Ketamine induced EP which is characterized by vivid dreams, hallucinations, illusions and delirium is thought to be related to reduced function of NMDAR, and serum glutamate and ketamine levels [11]. Younger age, higher dose, and longer duration of anesthesia significantly predicted EP occurrence and severity [6]. The symptoms were successfully eliminated with infusion of low dose midazolam without the need for other rescue medication. This neuroprotective effect of midazolam against glutamate toxicity occurs by increasing reuptake rate of GABA activity. This effect is demonstrable even at a low dose of midazolam, where in micro molar level inhibits $\mathrm{K}+, \mathrm{Na}+$, and $\mathrm{Ca}++$ dependent channels. That means midazolam confers neuroprotection in low dose by inhibiting positive ion channels in the presence of glutamate toxicity, but in high dose and longtime exposure induces neuronal apoptosis [12].

Majority of the past studies focused on the successful prevention strategy of delirium using concomitant midazolam and propofol presurgically [13]. Only haloperidol has been used for antagonistic effect on the receptor to prevent post operative delirium effect [5]. However, we have not found any report of the use of midazolam to revert delirium episode post treatment. Midazolam works by being hydroxylated by the cytochrome P450 (CYP) enzymes and directly enhances the effect of the GABA neurotransmitter. It affects the sensory inferences and perception. Midazolam in its metabolized form has a short half life. It also acts on glycine receptors and produces muscle relaxation effect. The use of midazolam premedication with ketamine had effectively attenuated the hemodynamic pressor response and postanesthetic emergence phenomenon $[14,15]$. Ketamine-induced cardiostimulatory effects may be blunted with concurrent administration of midazolam due to its known action on decreasing the peripheral vascular resistance thereby reducing the raised blood pressure. Midazolam also has anxiolytic, hypnosis, sedation and anti-epileptic property and has been widely used as adjunct to regional and local anaesthesia.

Emergence delirium can be provoked by stress leading to inappropriate response to auditory and visual stimuli. In an uncomplicated postoperative emergence, it may be attributable to the interference of neurotransmitter metabolism and function within the central nervous system causing hyper or hypostimulation of GABA receptor [16]. Another theory was related to specific transient paradoxical excitatory effect at locus coeruleus, as a result of GABAergic depolarization/excitation in the neocortical neurons leading to genesis of arousal and conciousness. However, the exact underlying mechanism of action associated with ketamine use that trigger emergence delirium which has yet to be fully understood and elucidated. 


\section{Conclusion}

Prolonged use and unmonitored ketamine dosage may cause long lasting delirium. Low intravenous dose midazolam is safe, helps patient anxiolytically and potentially reverses the delirium emergence symptoms.

\section{References}

[1] Wein S, Spruyt O, Chapman R. Ketamine as a possible cause of delirium. Journal of Pharmacy Practice and Research. 2002; 32(3):212-4.

[2] Hemmingsen C, Nielsen PK, Odorico J. Ketamine in the treatment of bronchospasm during mechanical ventilation. Am J Emerg Med. 1994;12:417-20.

[3] Corssen G, Revess J, Stanley T. Dissociative anesthesia. Anesthesia and Analgesia. Philadelphia, Lea \& Febiger, 1988; 89.

[4] Garfield JM, Garfield FB, Stone JG, Hopkins D, Johns LA. A comparison of psychologic responses to ketamine and thiopentalnitrous oxide-halothane anesthesia. Anesthesiology. 1972;36:329-38.

[5] Atlas GM, Milles M. Haloperidol for the treatment of ketamine-induced emergence delirium. J Anesth Clin Pharmacology. 2007;23(1):65-7.

[6] Gonsalves G, Baskaran D, Upadhyaya D. Prevention of emergence delirium in children - A randomized study comparing two different timings of administration of Midazolam. Anesth Essays Res. 2018;12(2): 522-7.

[7] Somashekara SC, Govindadas D, Devashankaraiah G, Mahato R, Deepalaxmi S, Srinivas $V$, et al. Midazolam premedication in attenuating ketamine psychic sequelae. Journal of Basic and Clinical Pharmacy. 2010; 1(4):209-13.

[8] Perumal DK, Adhimoolam M, Selvaraj N, Lazarus SP, Mohammed MA. Midazolam premedication for ketamine-induced emergence phenomenon: A prospective observational study. J Res Pharm Pract. 2015;4(2):89-93.

[9] Naldan ME, Taghizadehghalehjoughi A. neuroprotective effect of midazolam low dose against glutamate toxicity induced to olfactory, cortex and cerebellum neurons culture: In vitro study. Int J Med Res Health Sci. 2019;8(1):101-8.

[10] Jansen KL. A review of non medical use of ketamine: use, users and consequences. J Psychoactive Drugs. 2000;32:419-33.

[11] Stone JM, Erlandsson K, Arstad E, Squassante L, Teneggi V, Bressan RA, et al. Relationship between ketamine-induced psychotic symptoms and NMDA receptor occupancy: a [(123)I]CNS-1261 SPET study. Psychopharmacology. 2008;197:401-8.

[12] So EC, Wu KC, Kao FC, Wu SN. Effects of midazolam on ion currents and membrane potential in differentiated motor neuron-like NSC-34 and NG108-15 cells. European Journal of Pharmacology. 2014;724:152-60.

[13] Liu Y, Li X, Liang Y, Kang Y. Pharmacological prevention of postoperative delirium: $A$ systematic review and meta-analysis of randomized controlled trials. Evidence Based Complementary and Alternative Medicine. 2019;2019:9607129.

[14] Green SM, Krauss B. Clinical practice guideline for emergency department ketamine dissociative sedation in children. Ann Emerg Med. 2004;44(5):460-71.

[15] Lohit K, Srinivas V, Kulkarni C, Shaheen A clinical evaluation of the effects of administration of midazolam on ketamineinduced emergence phenomenon. J Clin Diagn Res. 2011;5:320-3.

[16] Visnath O, Kerner B, Jean YL, Soto R, Rosen G. Emergence delirium: a narrative review. J Anaesthesiol Clin Sci. 2015;4:2. 